\title{
A novel, automated SARS-CoV-2 saliva PCR test protects a global asymptomatic workforce
}

\author{
Nikki Carter
}

AstraZeneca (United Kingdom)

Maryam Clausen

AstraZeneca (Sweden)

Rebecca Halpin

AstraZeneca (United States)

Colin Blackmore

AstraZeneca (United Kingdom)

Kang Cai

AstraZeneca (United States)

Oona Delpuech

AstraZeneca (United Kingdom)

Alexander kohlmann

AstraZeneca (United States)

Otto Magnusson

AstraZeneca (Sweden)

Ruth March

AstraZeneca (United Kingdom)

Daniel O'Neill

AstraZeneca (United Kingdom)

Kasthuri Prakash

AstraZeneca (Sweden)

James Sherwood

AstraZeneca (United Kingdom)

Tabetha Sundin

AstraZeneca (United States)

Jason Swift

AstraZeneca (United Kingdom)

Azar Tarakameh

AstraZeneca (Sweden)

Marilou Wijdicks

AstraZeneca (United Kingdom)

Daniel Wise 
AstraZeneca (United Kingdom)

Mark Fidock (D mark.fidock@gmail.com)

AstraZeneca (United Kingdom)

\section{Research Article}

Keywords: PCR testing, SARS-CoV-2 virus, saliva sample

Posted Date: March 18th, 2021

DOl: https://doi.org/10.21203/rs.3.rs-246781/v2

License: (c) (1) This work is licensed under a Creative Commons Attribution 4.0 International License. Read Full License 


\section{Abstract}

Regular PCR testing of nasopharyngeal swabs from symptomatic individuals for SARS-CoV-2 virus has become the established method by which health services are managing the COVID-19 pandemic. Businesses such as AstraZeneca have also prioritised voluntary asymptomatic testing to keep workplaces safe and maintain supply of essential medicines to patients. We describe the development of an internal automated SARS-CoV-2 testing programme including the transformative introduction of saliva as an alternative sample type.

\section{Main}

Several organisations have responded to the challenge of keeping the workplace safe by implementing SARS-CoV-2 testing for asymptomatic individuals, either in their own laboratories or outsourced (https://www.gov.uk/government/publications/coronavirus-covid-19-testing-guidance-foremployers/annex-b-a-practical-guide-for-employers-who-want-to-offer-workplace-testing-forasymptomatic-employees; https://blog.aboutamazon.co.uk/working-at-amazon/how-we-ramped-uponsite-covid-19-testing-for-amazon-employees; https://www.bbc.co.uk/news/business-55370312). AstraZeneca has taken the additional step to increase adoption in a voluntary programme by operating automated SARS-CoV-2 testing, at scale and in an industrial context, using saliva as the sample type [Fig. 1].

In mid-March 2020, AstraZeneca set up an internal programme of voluntary SARS-CoV-2 testing for asymptomatic employees in UK, Sweden and US who could not work from home and were not able to obtain testing through their countries' national efforts. Launched in 18 days, this programme was focused on workers responsible for maintaining the company's supply of medicines, critical research and development laboratory staff and essential business employees. The initial launch of this effort used established nasopharyngeal swab (NPS) collection, supervised by a healthcare professional (HCP) (https://www.cdc.gov/coronavirus/2019-ncov/lab/guidelines-clinical-specimens.html). However, this proved to be uncomfortable and unpopular, leading to lower adoption than required. Following reports that saliva samples could be used in place of NPS for SARS-CoV-2 detection [1] we evaluated and subsequently implemented saliva as our preferred sample type [Fig. 2a \& 2b]. By February 2021 approximately 70,000 SARS-CoV-2 PCR tests have been completed within our three internal global testing centres, of which 54,000 are based on saliva.

Following the introduction of saliva testing, adoption by employees increased approximately 4-fold and over $90 \%$ of 1062 employees surveyed expressed a preference for the change [2].

Asymptomatic employees follow a 5-step process for SARS-CoV-2 testing:

1. Employee requests a test via a bespoke IT application that includes the employee recording potential symptoms for COVID-19 and any other information required by local laws. 
2. An HCP confirms that the individual is asymptomatic, has provided informed consent and orders the test.

3. Collection of the sample as advised by manufacturers' and local health authorities' instructions.

4. Analysis of sample for the presence of SARS-CoV-2 at one of three AstraZeneca laboratories: in Cambridge (UK), Gothenburg (Sweden) or Gaithersburg, MD (USA). The workflow was risk assessed prior to initiating employee testing and followed government guidance enabling the experimentation to be conducted at biosafety level 2 (BSL2), conforming to local laboratory standards including Good Clinical Practice (GCP) and Clinical Laboratory Improvement Amendments (CLIA) as appropriate. Positive results were reported at cycle threshold (Ct) values of $\leq 40$ and following the manufacturer's instructions.

5. Return of results by the HCP. Negative PCR results are reported in the IT app whereas the HCP contacts the employee to discuss the outcome of positive test results. The average turnaround time from sample receipt to result is $<24$ hours.

As expected, this testing cascade in asymptomatic individuals requires high analytic sensitivity. In our asymptomatic cohort, positive samples had a mean Ct value of 32 (Ct range of 40-15) consistent with reported values in similar populations [3]. Interestingly, although higher $\mathrm{Ct}$ values are associated with asymptomatic samples and lower infectiveness in viral cultures compared to samples from symptomatic cohorts, these differences are neither consistent nor statistically significant [4]. Recent publications have proposed that saliva collection reflects peak infection, predicts COVID-19 outcome and is associated with lower overall cost [5-7] compared to nasopharyngeal collection.

This project was facilitated by a bespoke IT application accessible via mobile phones, desktops or other computers. Test and sample data is linked to each AstraZeneca employee via their personal record identifier and a unique sample identity. The IT app ensures that the connection between this identity and sample is segregated and encrypted, while personally sensitive information is de-identified and highfidelity sample tracking is facilitated.

An average of $0.7 \%$ samples tested to date were positive in this voluntary internal programme, and individuals and their close contacts were advised to self-isolate according to national guidelines (https://www.ecdc.europa.eu/sites/default/files/documents/Guidance-for-discharge-and-ending-ofisolation-of-people-with-COVID-19.pdf; https://www.cdc.gov/coronavirus/2019-ncov/if-you-aresick/quarantine.html). Overall, this programme has achieved its objective of maintaining a safe workplace environment by keeping the infection rate approximately 10 -fold lower than that observed in the respective communities.

\section{Methods}

\section{Establishing asymptomatic testing}

a.genesig® COVID-19 Real-Time PCR (UK and Sweden) 
AstraZeneca laboratories in Europe used the genesig® COVID-19 Real-Time PCR assay (PrimerDesign) (PD) (https://www.fda.gov/media/136309/download), approved in Europe for use with NPS and saliva samples. This assay detects the ORF1ab gene and can detect 0.33 copies of whole viral genome RNA/ $\mu \mathrm{L}$, with a sensitivity of $\geq 95 \%$ and $100 \%$ specificity. The bioinformatic analysis of SARS-CoV-2 genomic epidemiology published on the GISAID EpiCoV ${ }^{\mathrm{Tm}}$ database is reviewed weekly by PD to verify that the assay is effective for new variants.

The assay was verified for linearity, reproducibility, user, temporal and instrument variability at AstraZeneca laboratories. Assessments were performed using SARS-CoV-2 RNA contrived samples (Twist Synthetic SARS-CoV-2 RNA (Control 1 and Control 2), Twist Bioscience, https://www.twistbioscience.com/resources/product-sheet/twist-synthetic-sars-cov-2-rna-controls), demonstrating that the assay was performing within acceptable limits as laid out in the instructions for use (IFU).

Linearity was shown to be high, with $93 \%$ of 14 experiments meeting the slope acceptance criteria (1.0 \pm 0.15 ) and $100 \%$ of 18 runs having an $R^{2} \geq 0.99$ and meeting the acceptance criteria of $\geq 0.95$. Limit of detection (LoD) was confirmed as 1 copy/ $\mu \mathrm{L}$ in $98 \%$ (41/42) of replicates, which is suitable for viral detection in asymptomatic individuals.

Intra-inter assay precision experiments indicated that the assay detected the contrived samples consistently. When an average $\mathrm{Ct}$ from replicate plates (same RNA extraction) was used for classification, $100 \%$ detection was observed for dilutions from 20-200,000 copies of RNA per well across 4 days of testing.

\section{b. TaqPath ${ }^{\mathrm{Tm}}$ RT-PCR COVID-19 Combo Kit (Thermo Fisher Scientific Inc.) (USA)}

In the AstraZeneca USA CLIA laboratory, the PD kit was not approved for use and available at the time of laboratory set up. Hence, assessments were performed using the TaqPath ${ }^{\text {TM }}$ RT-PCR COVID-19 Combo Kit (ThermoFisher Scientific Inc.) (TF) (https://www.fda.gov/media/137450/download). The TF Kit has specific target sequences for 3 genes: ORF1ab, N Protein, $S$ protein. According to the manufacturer, the LoD of the TF Kit is 10 copies of whole viral genome RNA, which will detect $\geq 95 \%$ positive samples.

Verification experiments used the same panel of Twist SARS-CoV-2 RNA contrived samples and successfully demonstrated assay linearity and dynamic range down to 10 copies per qRT-PCR reaction LoD. Reproducibility was demonstrated using spiked-in RNA level of 10 copies per reaction.

\section{Transition to saliva samples a. Sample collection}

Following a comparison of extraction and amplification efficiency for different saliva sample collection devices with appropriate regulatory approval, GeneFix ${ }^{\mathrm{TM}}$ (https://isohelix.com/wp- 
content/uploads/2020/12/GeneFix-brochure-2020_v6.pdf) was selected for use in Europe, and DNA Genotek OMNIgene ${ }^{\mathrm{TM}}$ (https://www.fda.gov/media/143416/download) in the US. These devices incorporate a funnel to facilitate saliva collection into a tube and contain a proprietary stabilisation buffer, enabling remote collection. Aligned to the instructions for use, employees were advised to be well hydrated and to fast for 30 minutes before sample collection [8]. Employees were supervised but selfcollected their saliva samples [9].

\section{b. Sample handling}

To simplify the protocol, the lysis buffer, proteinase $\mathrm{K}$ and the internal extraction control (IEC) could be premixed up to 2 hours in advance of use and added to a saliva sample simultaneously without impairing stability or sensitivity of RNA extraction.

\section{c. Clinical sensitivity}

20 paired saliva samples and NPS specimens were obtained from patients determined to be positive for SARS-Cov-2 by a separate qRT-PCR assay (AllPlex 2019-nCOV, https://www.fda.gov/media/137178/download) performed no more than 3 days prior to collection. Analysis demonstrated that the saliva sample was positive in every matched pair in which the NPS was positive [Fig. 2a].

\section{d. Analytical validity}

The analytical validity of saliva testing was evaluated through a comparison with NPS testing for impact on extraction, amplification and sensitivity and SARS-CoV-2 detection using contrived samples (Twist Bioscience [UK and Sweden]; Qnostics SARS-CoV-2 Analytical Q Panel 01, Cat \# SCV2AQP01-B [USA]).

The sensitivity for detecting SARS-CoV-2 was similar in contrived saliva and nasal samples [Fig. 2b]. In both cases assay failures were only seen at the lowest concentration and at comparable rates. Saliva did not impact extraction or amplification.

\section{e. Optimisation of saliva testing}

Saliva testing protocols were optimised as follows to ensure a low intrinsic test failure rate. This resulted in a failure rate of $<0.5 \%$ of samples per run.

\section{i. Sample volume}

Input volumes between $50 \mu \mathrm{L}$ and $600 \mu \mathrm{L}$ were evaluated, $600 \mu \mathrm{L}$ limited RNA binding to the Beckman Coulter beads during extraction whereas $50 \mu \mathrm{L}$ resulted in increased assay failures (https://www.beckman.com/search\#q=C58529AA\&t=coveo-tab-techdocs). The input volume for both PCR tests was standardised at $200 \mu \mathrm{L}$.

\section{ii. Duration of reverse transcription}


Extending the reverse transcription time to 30 minutes for saliva samples $(\mathrm{N}=8)$ reduced assay failure and improved sensitivity (Ct values), although the effect size was relatively small.

\section{iii. Automation}

The introduction of automated sample handling (Biomek i5 and i7 liquid handlers for transfer from collection tube to plate, lysis and heat inactivation and magnetic bead extraction of RNA, https://www.beckman.com/landing/ppc/liquid-handlers/biomek/i-series) reduced processing time by around $20 \%$ and reduced test failures 10 -fold, from 5-0.5\%, compared to manual testing.

\section{iv. Addition of agents}

One of the major challenges in the transition to automated testing of saliva samples was sample viscosity. The addition of $50 \mu \mathrm{L}$ of $1 \mathrm{M}$ dithiothreitol (DTT, Sigma Aldrich) to $2 \mathrm{ml}$ saliva plus stabiliser reduced sample viscosity and improved automated tube to plate transfer. Addition of DTT did not affect test sensitivity of either synthetic contrived or clinical SARS-CoV-2 samples.

\section{Declarations}

\section{Acknowledgements}

The authors would like to acknowledge the following for their contributions to the testing programme: Daisy Belfield, Graham Belfield, Martin Booth, Helen Brown, Benjamin Guscott, Linda Kitching, Ingela Maxvall, Patrick O’Shea, Marta Potapo, Katya Ribeiro Passos, Jonathan Sumner, Paul Turner, Abhishek Upadhyay, Liisa Vilén,

\section{Author Contributions}

Each author has made substantial contributions to the conception and design of the work, or the software used, including data analysis and interpretation. All authors have contributed to the draft and have reviewed the submitted manuscript.

\section{Study Participation and Methods}

Informed consent was obtained from all participants within this internal SARS-CoV-2 testing program, consent was reviewed by the AstraZeneca Bioethics Advisory Board. This study was approved by the AstraZeneca internal review body with all methods performed in accordance with local relevant guidelines and standard operating procedures from AstraZeneca. Sourced samples for methods development were obtained according to AstraZeneca Human Biosamples policy, which includes review of informed consent and ethics approvals.

\section{Competing Interests statement}

All authors are employees of AstraZeneca and have financial interests in the company. 


\section{References}

1. Wyllie, A. L. et al. Saliva or Nasopharyngeal Swab Specimens for Detection of SARS-CoV-2. Engl. J. Med. 383, 1283-1286 (2020)

2. Romo M. et al. Patterns of SARS-CoV-2 testing preferences in a national cohort in the United States. Preprint at https://www.medrxiv.org/content/10.1101/2020.12.22.20248747v1.full (2020).

3. Zhou, R. et al. Viral dynamics in asymptomatic patients with COVID-19, Int J Infect Dis 96, 288-290, (2020)

4. Cereda, D. et al. The early phase of the COVID-19 outbreak in Lombardy, Italy. Preprint at https://arxiv.org/abs/2003.09320 (2020).

5. To KK-W. et al. Temporal profiles of viral load in posterior oropharyngeal saliva samples and serum antibody responses during infection by SARS-CoV-2: an observational cohort study. Lancet Infect Dis; 20, 565-74. (2020).

6. Silva, J. et. Al. Saliva viral load is a dynamic unifying correlate of COVID-19severity and mortality. Preprint at https://www.medrxiv.org/content/10.1101/2021.01.04.21249236v2 (2021).

7. Bastos, et. al. The Sensitivity and Costs of Testing for SARS-CoV-2 Infection With Saliva Versus Nasopharyngeal Swabs. Ann Intern Med doi: 10.7326/M20-6569 (2021).

8. Landry, M. et al, Challenges in use of saliva for detection of SARS CoV-2 RNA in symptomatic outpatient. J Clin Virol 130, 104567 (2020).

9. Byrne, R. L. et al. Saliva offers a sensitive, specific and non-invasive alternative to upper respiratory swabs for SARS-CoV-2 diagnosis. Preprint at https://www.medrxiv.org/content/10.1101/2020.07.09.20149534v1 (2020).

\section{Figures}




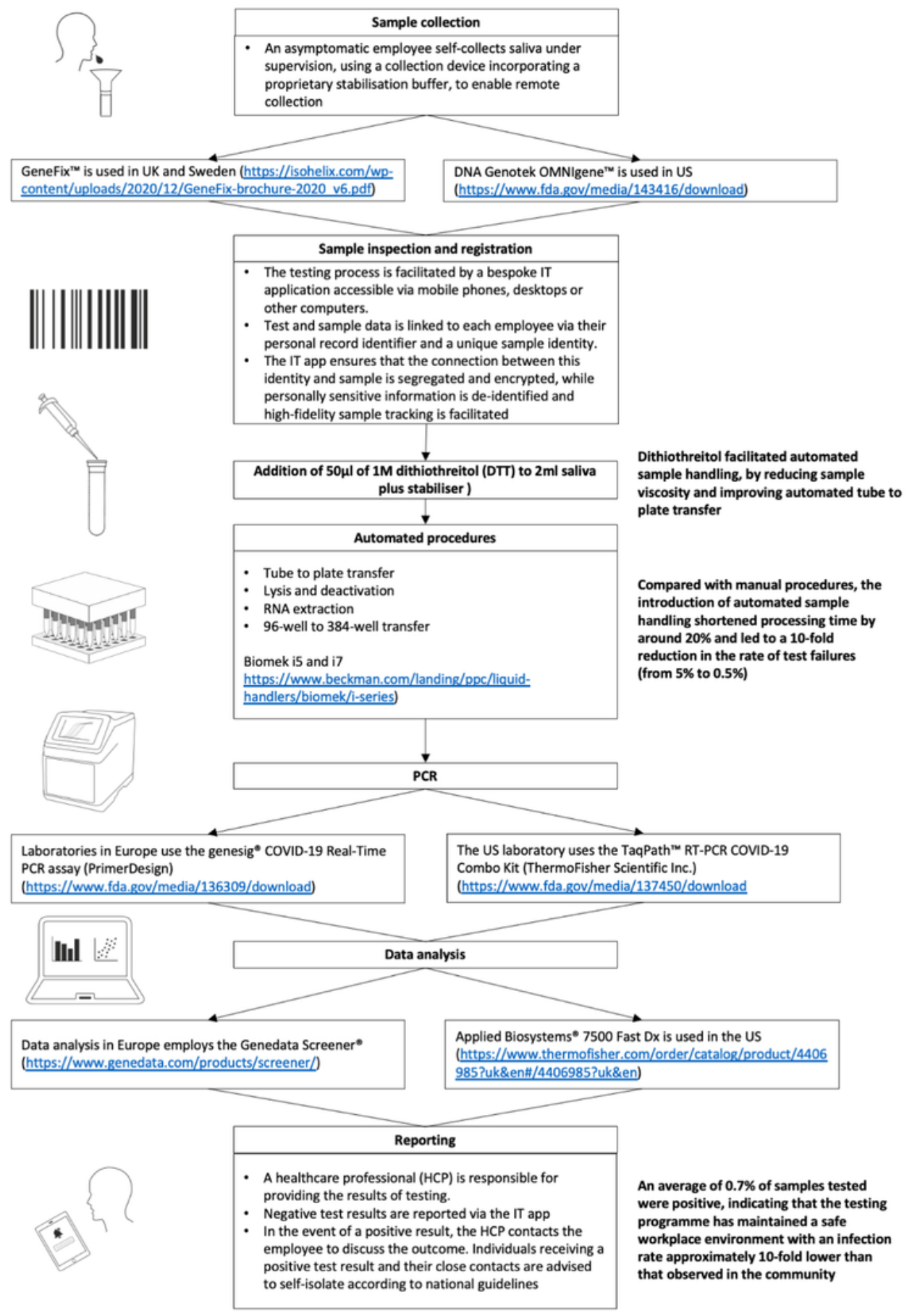

\section{Figure 1}

Overview of the automated workflow for SARS-CoV-2 testing of asymptomatic employees. Saliva samples are tested at one of three AstraZeneca laboratories: in Cambridge (UK), Gothenburg (Sweden) or Gaithersburg, MD (USA). The workflow was risk assessed and follows government guidance enabling the experimentation to be conducted at biosafety level 2 (BSL2), conforming to local laboratory standards 
including Good Clinical Practice (GCP) and Clinical Laboratory Improvement Amendments (CLIA) as appropriate.

a

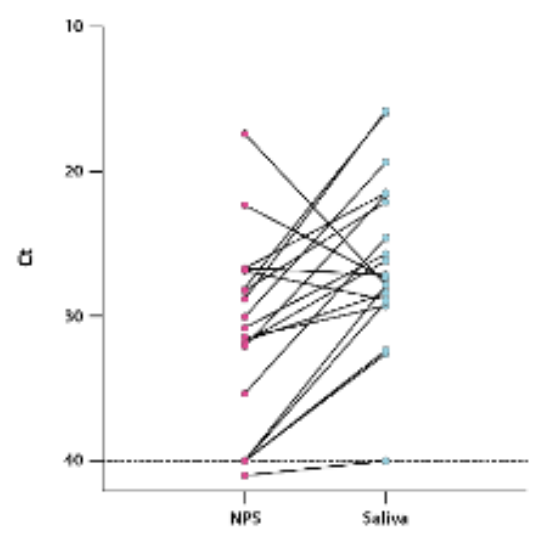

b
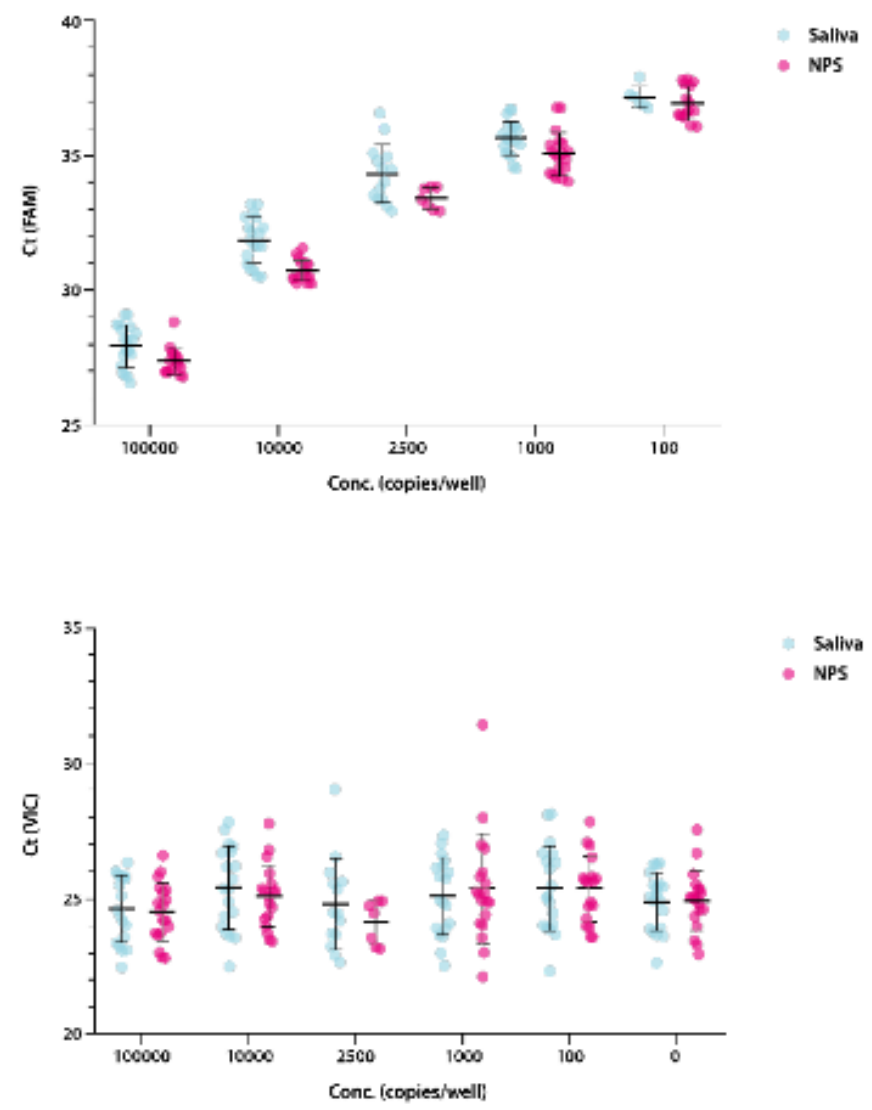

Figure 2

Clinical and analytical validation of the SARS-CoV-2 PCR tests enabling the transition from NPS to saliva. a, Analysis of 20 paired clinical saliva samples and NPS tested using the TF assay. Ct values for all samples/detectors were generated using the Design and Analysis Software version 2.4.3 (ThermoFisher), 
equivalent results seen using the PD assay (data not shown). b, Comparison of the analytical sensitivity of testing saliva samples and NPS, conducted on contrived samples using the PD assay. The sensitivity for detecting SARS-CoV-2 was similar for both sample types (FAM), and sample type had no effect on extraction or amplification (VIC). 